\title{
Contributo para a adaptação transcultural e validação da «Breastfeeding Self-Efficacy Scale - Short Form» - versão portuguesa
}

Vanessa Santos, ${ }^{*}$ Sónia Bárcia**

\section{RESUMO}

A amamentação apresenta muitas vantagens, tanto para a mãe, como para o bebé. É reconhecida pelas organizações internacionais como a OMS/UNICEF/Academia Americana de Pediatria como o melhor método para alimentar uma criança. Contudo, muitas mães abandonam a amamentação precocemente. Nesse sentido é necessário identificar os factores responsáveis por este facto. Os instrumentos de medida são uma boa ferramenta de avaliação ao dispor dos profissionais de saúde. A «Breastfeeding Self-Efficacy Scale - Short Form (BSES-SF)» é um instrumento de auto-preenchimento composto por 14 itens concebidos para avaliar a confiança das mães na amamentação. O objectivo deste estudo é dar um contributo na validação da BSES-SF, para a realidade portuguesa. Foram realizados os procedimentos para a validade de conteúdo, consistência interna e estabilidade do instrumento ao longo do tempo. Na avaliação das propriedades psicométricas, foi analisada a validade de conteúdo por um comité de sete peritos. A consistência interna e o teste/re-teste foram medidos utilizando uma amostra constituída por 31 puérperas a amamentar, que preencheram a escala na $1^{\mathrm{a}}-2^{\mathrm{a}}$ semana após o parto e novamente uma semana mais tarde. Em relação à fiabilidade, a consistência interna da BSES-SF deu um valor de 0,95 no coeficiente alfa de Cronbach e um valor de 0,52 na reprodutibilidade teste/re-teste. Nas sete questões secundárias levantadas, verificou-se que a relação entre a auto-eficácia e experiência anterior de amamentação, aleitamento materno exclusivo e a amamentação imediatamente após o parto, são estatisticamente significativos. As habilitações literárias, estado civil, idade e tipo de parto não influenciaram significativamente a auto-eficácia. Este estudo revela que a versão traduzida da BSES-SF é uma medida válida e fiável de auto-eficácia na amamentação.

Palavras-Chave: Amamentação; Instrumentos de Medida; Adaptação Cultural; Validação; Breastfeeding Self-Efficacy Scale.

\section{INTRODUÇÃO}

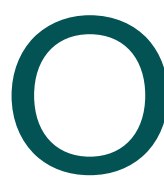
aleitamento materno é considerado um dos pilares fundamentais para a promoção e protecção da saúde das crianças em todo o mundo. A sua prevalência tem sido muito variável ao longo dos tempos, tendo atingido valores muito reduzidos nas décadas que se seguiram à II Guerra Mundial, devido às alterações sociais e comportamentais que modificaram o estilo de vida das mulheres. Após os anos 70 verificou-se um retorno gradual à

*Licenciada em Fisioterapia

**Docente do Curso de Fisioterapia, Universidade Atlântica prática do aleitamento materno, sobretudo nas mulheres mais informadas. Nos últimos anos, através dos esforços da Organização Mundial de Saúde (OMS) e de muitas outras entidades, têm-se realizado estudos para averiguar as causas deste declínio e estabelecer estratégias que conciliem a prática do aleitamento materno com as condições de vida moderna. ${ }^{1}$

Em Portugal, os estudos epidemiológicos parecem confirmar que as taxas de amamentação têm tido uma melhoria progressiva nas últimas duas décadas. ${ }^{1,2} \mathrm{Os}$ dados nacionais situam-se dentro do intervalo de variação dos países do Sul da Europa, onde as taxas de iní- 
cio de amamentação se aproximam dos $80 \%$, rondam os $50 \%$ aos três meses e decrescem para os $25 \%$ aos seis meses. $^{2}$

O sucesso da amamentação depende de muitos factores. $\mathrm{O}$ apoio por parte do companheiro é fundamental, a mãe fica mais confiante na escolha de amamentar se o parceiro estiver de acordo e confiante em relação à amamentação. ${ }^{3,4}$ A restante família e os amigos também podem ser fonte de encorajamento, ou pelo contrário contribuir para o aumento das dificuldades. Como acontece às avós que não amamentaram pela chamada «síndrome do leite insuficiente», que têm tendência a questionar a quantidade e qualidade do leite e a criar um ambiente adverso à amamentação. ${ }^{5}$ Também as práticas da maternidade, nomeadamente a adaptação do recém-nascido ao seio, de preferência na primeira meia hora, são importantes para iniciar a amamentação e a vinculação mãe-filho. ${ }^{6,7}$

Entre as razões subjectivas mais frequentes para o insucesso da amamentação, conta-se a circunstância de muitas mães acreditarem que não têm leite suficiente, em quantidade ou qualidade, ou terem tido anteriormente dificuldade em amamentar. ${ }^{8}$ No âmbito fisiológico, os problemas que ocorrem mais frequentemente são o ingurgitamento mamário, as mastites, que podem ser dolorosas, as gretas ou fissuras nos mamilos, os mamilos dolorosos, a insuficiência quantitativa da produção de leite e a deficiência qualitativa do leite. Em todas estas situações, a solução passa, normalmente, pela continuação da amamentação, em posição apropriada e com um procedimento técnico adequado. $^{9}$

Recentemente a questão da auto-eficácia tem sido vista como um excelente modo de prever os comportamentos relacionados com a saúde. Identificada como a percepção que um indivíduo tem de que é capaz de desempenhar uma tarefa ou comportamento específico, a auto-eficácia compõe-se de duas partes: (a) expectativas de resultados, a percepção de que um determinado comportamento gera um determinado resultado; e (b) expectativas da auto-eficácia, a convicção do indivíduo de que é capaz de realizar uma tarefa ou comportamento específicos para obter o resultado desejado. Esta distinção é importante. As pessoas podem acreditar que um determinado comportamento as irá ajudar a atingir um determinado resultado, mas estarem pouco confiantes de que irão desempenhar esse comportamento numa situação específica. Por exemplo, se uma mãe acreditar que é importante não introduzir uma fórmula láctea suplementar, mas não tiver confiança na sua capacidade de manter a produção de leite ou de determinar se o bebé recebe leite materno suficiente, torna-se provável que essa mãe vá introduzir o suplemento lácteo. ${ }^{10,11}$

Estas expectativas de auto-eficácia influenciam os comportamentos do indivíduo de várias formas: o esforço que despende, durante quanto tempo persiste quando enfrenta obstáculos, ou se se deixa envolver em processos cognitivos auto-debilitantes ou encorajadores. Assim, a auto-eficácia na amamentação é uma variável importante para os resultados do aleitamento, uma vez que prevê: (a) se a mãe opta por amamentar ou não, (b) o grau de esforço investido no processo, (c) se a mãe apresenta padrões de pensamento auto-debilitantes ou de auto-elevação, e (d) de que forma reage emocionalmente às dificuldades da amamentação. ${ }^{10,11}$

Na auto-eficácia existem quatro fontes principais de informação: (a) resultados de desempenhos (experiências anteriores de amamentação), (b) experiências visuais (observação do desempenho nesse comportamento), (c) persuasão verbal (encorajamento por parte de pessoas significativas), e (d) reacções fisiológicas (reacções somáticas à consciencialização autónoma durante a antecipação ou experiência de um acontecimento potencialmente desgastante). Em relação à auto-eficácia na amamentação, uma mãe determina a sua capacidade de amamentar o recém-nascido com base em experiências anteriores de amamentação, na observação de comportamentos de amamentação de outras mães e no encorajamento por parte das pessoas que influenciam a sua vida. Também, o seu estado psicológico e afectivo, incluindo fadiga, stress ou ansiedade, é uma importante fonte de informação para avaliar a sua capacidade de amamentar. ${ }^{11}$

Foram realizados vários estudos para identificar as mães em risco de abandonar precocemente a amamentação, mas muitos dos factores de prognóstico são variáveis demográficas que não podem ser modificadas, como a idade da mãe, estado civil, nível de escolaridade e nível socioeconómico. ${ }^{12}$

O efeito da confiança materna nos resultados da amamentação tem sido salientado por diversos inves- 
tigadores. Num estudo transversal de 64 mulheres nos EUA, conclui-se que as grávidas sem confiança nas suas capacidades de amamentar iriam provavelmente desistir antes de 2 semanas após o parto (rácio de risco $=2,38$; $95 \%$ intervalo de confiança $[\mathrm{IC}]=1,82-6,18)$. De igual modo, descobriu-se que $27 \%$ das mulheres com uma baixa confiança, antes do parto, na sua capacidade de amamentar, desistiam de amamentar durante a primeira semana, em comparação com apenas $5 \%$ das mulheres com confiança elevada ( $\mathrm{p}<0,001)$. O insucesso na amamentação é 4 a 5 vezes mais provável em mães menos confiantes. ${ }^{11}$

A evidência científica actual aponta para o facto de que todas as formas de apoio, profissional ou outro, aumentam a duração do aleitamento materno (AM). O apoio profissional parece aumentar especialmente a duração do AM exclusivo, enquanto o apoio por leigos parece ser eficaz em todos os tipos de AM. Há ainda evidência de que a formação das equipas de saúde hospitalares segundo o modelo da OMS/UNICEF contribui significativamente para o prolongamento do AM exclusivo. $^{13}$

\section{MÉTODOS}

\section{Amostra}

A amostra é constituída por 31 puérperas seguidas nos Centros de Saúde da Parede e de Cascais, no período de Julho a Outubro de 2008. Os critérios de inclusão foram: utentes a amamentar na primeira ou segunda semana de vida do bebé, mães com idade igual ou superior a 18 anos, mães que frequentem os Centros de Saúde da Parede e de Cascais e que compreendam a língua portuguesa. Como critérios de exclusão: bebés com patologia que os impossibilite de mamar, utentes a amamentar na terceira semana de vida do bebé, mães com patologia que desaconselhe a amamentação, mães em aleitamento materno exclusivo mas por biberão, e mães que vão deixar de amamentar no momento da primeira ou segunda aplicação da escala.

As trinta e uma utentes dos Centros de Saúde de Cascais e da Parede que participaram na investigação têm uma média de idades de 30,8 anos $(\mathrm{dp}=4,5)$, a utente mais nova tem 22 anos e a mais velha 41 anos. O escalão etário modal situa-se no intervalo 31-35 anos, que compreende $48,4 \%$ do total das inquiridas. A maioria das inquiridas tem o ensino superior $(51,6 \%)$, seguin- do-se as utentes com o ensino secundário (42\%) e as que têm o ensino básico $(6,4 \%)$. Em termos de estado civil, predominam as casadas (68\%) e as utentes em união de facto (29\%). Cerca de $48 \%$ são primíparas, $42 \%$ têm o segundo filho e 9,7\% o terceiro. No que se refere ao tipo de parto, constata-se que a generalidade se refere a partos de cesariana com epidural $(35,5 \%)$, seguindo-se os partos eutócicos (29\%). Mais de metade da amostra já tinha tido experiência anterior de amamentação (52\%) e o tipo de amamentação mais praticado é o aleitamento materno exclusivo $(74,2 \%)$.

\section{Instrumento \\ Breastfeeding Self-Efficacy Scale - Short Form (BSES-SF)}

A Breastfeeding Self-Efficacy Scale (BSES), desenvolvida em 1999 por Cindy-Lee Dennis e Sandra Faux, é uma escala que identifica as mães que amamentam no período pós-parto imediato e que necessitam de suporte adicional. Identifica a confiança das mães na amamentação: mães em risco de abandono precoce, comportamentos e cognições relacionadas com a amamentação, individualização de estratégias de confiança, avaliação das intervenções e desenvolvimento de um programa guião. ${ }^{10} \mathrm{Em}$ 2003, esta versão foi revista por Cindy-Lee Dennis e desenvolvida uma Short-Form da BSES. Assim, a BSES passou de 33 items para a BSES-SF, com 14 items, cujas propriedades psicométricas foram estudadas numa amostra de 491 mães, sendo cada pergunta respondida segundo uma escala de Likert de um a cinco: 1 - nada confiante, 2 - pouco confiante, 3 - às vezes confiante, 4 - confiante, 5 - muito confiante. ${ }^{10,11} \mathrm{Em}$ anexo será apresentada a versão portuguesa da BSES-SF (Anexo 1).

\section{Procedimentos}

O estudo efectuado é uma adaptação transcultural e validação de um instrumento de medida na área da saúde. O objectivo principal é traduzir, adaptar e validar para a cultura portuguesa o instrumento de medida «Breastfeeding Self-Efficacy Scale - Short Form», em termos de validade de conteúdo e mantendo a fiabilidade (validade interna através do alfa de Cronbach e reprodutibilidade teste/re-teste). O objectivo secundário é tentar perceber qual a influência da auto-eficácia na amamentação de sete factores: a idade, a experiência 
anterior de amamentação, as habilitações literárias, o estado civil, o tipo de parto, ter ou não amamentado logo após o parto e o tipo de amamentação.

A adaptação inter-cultural de um instrumento envolve dois passos principais: avaliação das equivalências conceptuais e linguísticas e avaliação das propriedades psicométricas. Segundo o European Group on Health Outcomes (ERGHO) estes são os critérios que permitem considerar que determinada medida tem equivalência cultural. ${ }^{14}$

A avaliação das equivalências conceptual e linguística inicia-se com um processo de tradução. Esta deve ser realizada por dois ou mais tradutores independentes, bilingues e multi-profissionais, ou tradutores oficiais cuja língua-mãe é a da versão a adaptar. A versão assim obtida deve ser retrovertida para a língua original por tradutores cuja língua-mãe seja a da versão original. As duas versões na língua original devem então ser comparadas. ${ }^{14}$

A adaptação inter-cultural de um instrumento envolve duas fases principais: (1) avaliação das equivalências conceptuais e linguísticas e (2) avaliação das propriedades psicométricas.

(1)

- Foi feito um pedido formal à autora para a tradução da escala, o qual foi autorizado.

- Tradução e retroversão cegas: realizadas por 2 tradutores.

- As versões de consenso da tradução e retroversão foram feitas pelas autoras deste artigo.

- A versão final da retroversão foi aprovada pela autora da escala.

(2)

- Avaliação por um painel de sete peritos, três fisioterapeutas, duas médicas e duas enfermeiras, da versão portuguesa da BSES-SF.

- O painel avaliou quanto ao formato, apresentação, realidade portuguesa e nível de compreensão.

- Critérios de inclusão para os sete peritos: experiência na área da saúde da mulher ou de aleitamento materno, mínimo de seis anos de experiência e grau académico de licenciatura.

Após estas etapas foi efectuado um pré-teste a um pequeno grupo de puérperas. Passando em seguida à aplicação da escala em contexto real, foi feita em dois tempos: um primeiro com acompanhamento de uma das au- toras, na primeira ou segunda semana de vida do bebé e um segundo tempo, uma semana após a primeira.

\section{RESULTADOS E DISCUSSÃO}

Análise das dimensões da escala

Os valores descritivos da escala encontram-se apresentados no Quadro I, assim como, o respectivo Alfa de Cronbach.

QUADRO I. Média, Desvio Padrão e Alfa da Escala

\begin{tabular}{|lccc} 
Escala & Média & Desvio-Padrão & Alfa \\
\hline BSES-SF & 53,4 & 12,2 & 0,95 \\
\hline
\end{tabular}

O Alfa de Cronbach da versão portuguesa foi de 0,95, semelhante ao da versão original, que foi de 0,94 e acima do da versão polaca que foi de 0,89 . A média que se obteve é muito semelhante aos valores obtidos pela autora na escala original $(55,8)$ e o valor do desvio-padrão da BSES-SF foi ligeiramente superior do que na escala original $(10,8)$.

A consistência interna da escala de auto-eficácia da amamentação (BSES-SF) foi avaliada com recurso ao coeficiente de consistência interna Alfa de Cronbach. O valor obtido na versão portuguesa da BSES-SF foi de 0,95. Segundo Hill e Hill (2005), quando se obtêm valores de alfa de Cronbach acima de 0,9, este pode ser considerado excelente. ${ }^{15}$

- >0,9- Excelente

- 0,8-0,9-Bom

- 0,7-0,8-Razoável

- 0,6-0,7- Fraco

- < 0,6 - Inaceitável

\section{REPRODUTIBILIDADE (TESTE/RETESTE)}

QUADRO II. Correlação entre tempo 1 e tempo 2

BSES-SF 2

BSES-SF 1 0,52

$p<0,001$

O coeficiente de reprodutibilidade (teste/reteste) medido através do coeficiente de correlação momento- 
produto de Pearson entre a primeira aplicação e a segunda aplicação é de $(\mathrm{r}=0,52)$, diferente do da escala original na $1^{\mathrm{a}}(r=0,99), 4^{\mathrm{a}}(r=0,99)$ e $8^{\mathrm{a}}(r=0,99)$ semanas após o parto. Segundo Pestana e Gageiro (2005) este valor pode ser considerado positivo, moderado e significativo $^{16}$

$-<0,2$ - Muito fraca

- 0,2-0,4-Fraca

- 0,4-0,7 - Moderada

- 0,7-0,9-Elevada

- >0,9- Muito elevada

\section{Questões secundárias}

Uma vez que a auto-eficácia pode ser influenciada por diversos factores, fomos ainda tentar perceber qual a relação entre sete factores: a idade, a experiência anterior de amamentação, as habilitações literárias, o estado civil, o tipo de parto, ter ou não amamentado logo após o parto e o tipo de amamentação, na auto-eficácia da amamentação.

Nas questões secundárias levantadas, é utilizada como referência, para aceitar ou rejeitar a hipótese nula, um nível de significância $(\alpha) \leq 0,05$. Nas questões 1,2,3 como foram comparados dois grupos em variáveis dependentes medidas em escalas de rácio, utilizou-se o teste $t$ de Student para amostras independentes.

Questão 1 - Será que a experiência anterior de amamentação influencia significativamente a auto-eficácia da amamentação?

Questão 2 - Será que as mulheres que amamentaram o bebé logo após o parto obtêm valores superiores na escala de amamentação aos das mulheres que não amamentaram logo o seu bebé?

Questão 3 - Será que o tipo de amamentação influencia significativamente a auto-eficácia da amamentação?

Questão 1

A experiência anterior de amamentação influenciou significativamente a auto-eficácia da amamentação, pois $\mathrm{t}(29)=-2,319, \mathrm{p}=0,028$. As mulheres com experiência anterior de amamentação obtêm valores mais elevados na escala de auto-eficácia da amamentação do que as mulheres sem experiência anterior de amamentação $(\mathrm{m}=58,0$ versus 48,5$)$.

\section{Questão 2}

As mulheres que amamentaram o bebé logo após o parto obtêm valores significativamente superiores na escala de auto-eficácia de amamentação aos das mulheres que não amamentaram logo o seu bebé $(m=56,7$ versus 46,5$)$, com $\mathrm{t}(29)=2,345, \mathrm{p}=0,026$

\section{Questão 3}

A relação é estatisticamente significativa pois $t(29)=$ $6,822, p=0,000$. As mulheres que amamentam os bebés com leite materno exclusivo obtêm valores significativamente superiores na escala de auto-eficácia de amamentação aos das mulheres que fazem aleitamento misto $(\mathrm{m}=58,9$ versus 37,5$)$.

Estes resultados, obtidos estatisticamente, são consistentes com as mais antigas tradições da humanidade e com os avanços mais recentes da ciência. Apesar de as habilitações literárias, estado civil, idade e tipo de parto não influenciarem significativamente a auto-eficácia neste estudo, pois obtiveram $\mathrm{p} \geq 0,05$, tal como nos resultados da escala original (com excepção do tipo de parto, factor que não é contemplado na escala original), estes constituem bons indicadores, que possivelmente numa amostra maior se alterariam e poderiam vir a influenciar a amamentação.

No âmbito da actual política de internamentos de curta duração no hospital, a BSES-SF fornece aos profissionais de saúde um instrumento clínico útil.

\section{Limitações/Restrições}

As pequenas dimensões da amostra (31 mães) deveram-se às limitações temporais e de recursos humanos. Como pode observar-se na descrição da amostra esta é muito homogénea, pelo que não é representativa das mulheres portuguesas. Como tal, não se obteve uma variabilidade suficiente em algumas respostas ao questionário, de modo a detectarem-se diferenças na auto-eficácia da amamentação.

\section{CONCLUSÃO}

O objectivo deste trabalho foi o de dar um contributo para a adaptação e validação cultural do instrumento de medida BSES-SF para a realidade portuguesa. Este propósito foi atingido, contudo é de salientar que a amostra utilizada foi de conveniência e de dimensões reduzidas. Este instrumento pretende avaliar a confian- 
ça das mães durante a amamentação.

No final das várias etapas metodológicas, constatou-se que os resultados encontrados na amostra utilizada nesta investigação psicométrica, foram consistentes com o estudo original e revelam que a versão portuguesa da BSES-SF pode ser um valioso instrumento de medição da confiança das mães na amamentação. Em relação à validade de conteúdo, embora não tenha havido uma concordância total entre peritos, pode-se salientar que houve um bom nível de consenso, tendo-se introduzido pequenas alterações a nível linguístico e cultural. $\mathrm{O}$ valor da validade interna foi considerado excelente pois encontra-se acima de 0,9 e o coeficiente de reprodutibilidade pode ser considerado positivo, moderado e significativo, pois foi de 0,52 .

Uma vez que se encontraram relações estatisticamente significativas entre a BSES-SF e a experiência anterior de amamentação, a amamentação logo após o

\begin{tabular}{lc}
\hline \multicolumn{2}{l}{ QUADRO III. Questões Secundárias significativas } \\
\begin{tabular}{lc} 
& BSES-SF \\
\hline Experiência anterior de amamentação & $P=0,028$ \\
Amamentação logo após o parto & $P=0,026$ \\
Tipo de amamentação & $P=0,000$
\end{tabular}
\end{tabular}

parto e o tipo de amamentação, seria importante realizar novos estudos com diferentes amostras no sentido de perceber se estas associações se mantêm.

A BSES-SF é uma preciosa ferramenta ao dispor dos profissionais de saúde no sentido de identificar mães em risco de abandonarem precocemente o acto de amamentar, avaliar comportamentos inerentes à amamentação, avaliar a eficácia das intervenções ou ajudar na construção de programas direccionados à díade mãe-bebé.

\section{REFERÊNCIAS BIBLIOGRÁFICAS}

1. Sarafana S, Abecasis F, Tavares A, Soares I, Gomes A. Aleitamento materno: evolução na última década. Act Ped Port 2006 Jan-Fev; 37 (1): 9-14 .

2. Cardoso L. Aleitamento Materno uma prática de educação para a saúde no âmbito da enfermagem obstétrica [Dissertação]. Braga: Universidade do Minho; 2006. p. 110.

3. LLLI. La Leche League Portugal. Disponível em: http://www.llli.org/Portugal.html [acedido em 19/10/2009].

4. NCT: Information Sheet: Fathers and Breastfeeding. [Internet]. National Childbirth Trust 2008. Disponível em: http://www.nct.org.uk/info-centre/publications/view/37 [acedido em 22/10/2008].

5. Portal da Saúde. Site do Aleitamento Materno. Disponível em: http://www.amamentar.net/ [acedido em 25/10/2008].

6. Michaelsen K, Weaver L, Branca F, Robertson A. Feeding and nutrition of infants and young children. Geneva:WHO; 2003.

7. Kennell H, Klaus $H$. Vínculo afetivo: observações recentes que alteram o cuidado perinatal. Disponível em: Available from http://www.ibfan.org.br/documentos/mes/doc4_99.pdf [acedido em 12/09/2008].

8. Coutinho J, Leal IP. Atitudes de mulheres em relação à amamentação: Estudo exploratório. Anál Psicol 2005 Jul; 23 (3): 277-82.

9. Galvão D. Amamentação bem sucedida: alguns factores determinantes. Loures: Lusociência; 2006.

10. Dennis CL, Faux S. Development and Psychometric Testing of the Breastfeeding Self-Efficacy Scale. Res Nurs Health 1999 Oct; 22 (5): 399-409.

11. Dennis CL. The breastfeeding self-efficacy scale: psychometric assessement of the short form. J Obstet Gynecol Neonatal Nurs 2003 Nov-Dec; 32 (6): 734-44.

12. Dennis CL. Identifying predictors of breastfeeding self-efficacy in the immediate postpartum period. Res Nurs Health 2006 Aug; 29 (4): 256$-68$.

13. Mama Mater: Associação Pró-Aleitamento Materno em Portugal. Newsletter 2008. Disponível em: http://www.mamamater.org/newsletter.html [acedido em 19/10/2008].

14. Ferreira PL, Marques FB. Adaptação cultural e linguística In: Ferreira PL, Marques FB, compiladores. Avaliação psicométrica e adaptação cultural e linguística de instrumentos de medição em saúde: princípios metodológicos gerais. Coimbra: Centro de Estudos e Investigação em Saúde da Universidade de Coimbra; 1998. p. 19-20.

15. Hill MM, Hill A. Investigação por questionário. $2^{a}$ ed. Lisboa: Edições Sílabo; 2005.

16. Pestana MH, Gajeiro JN. Análise de dados para ciências sociais: a complementaridade do SPSS. $5^{a}$ ed. Lisboa: Sílabo; 2008.

Conflitos de Interesse: não assinalados

\section{ENDEREÇO PARA CORRESPONDÊNCIA \\ Vanessa Santos \\ E-mail: nexasantos@gmail.com}




\begin{abstract}
Breastfeeding has many advantages for both the mother and the baby. It is recognized by international organizations such as WHO / UNICEF / American Academy of Paediatrics as the best method to feed a child. Many mothers prematurely discontinue breastfeeding. So it is very important to identify the factors which are responsible for this. Measurement Instruments are good assessing tools for health professionals. Breastfeeding Self-Efficacy Scale - Short Form (BSES-SF) is an auto fill measure instrument composed by 14 items meant to evaluate mothers' confidence in breastfeeding. The main purpose of our study is to contribute to validate the BSES-SF to the Portuguese reality. Procedures were made to construct validity, internal consistency and instrument's stability in time. A seven expert Committee analyzed the construct validity. Internal consistency and test/ re-test were measured using a sample of 31 breastfeeding mothers, who filled the scale in the $1^{\text {st }}-2^{\text {nd }}$ week postpartum and again one week later. BSES-SF internal consistency showed a Cronbach's alpha coefficient of 0,95, and 0,52 in test/ re-test. Concerning seven secondary questions about factors which could influence self-efficacy in breastfeeding, we could verify statistical significance between self-efficacy and previous breastfeeding experience, exclusive breastfeeding, and breastfeeding immediately after birth. Educational level, marital status, age and the kind of birth had no significant influence in self-efficacy. This study showed that the translated version of BSES-SF is a valid and reliable measure of self-efficacy in breastfeeding.
\end{abstract}

Keywords: Breastfeeding; Measure instruments; Cross adaptation; Validation; Breastfeeding Self-Efficacy Scale.

\title{
ANEXO \\ Breastfeeding Self-Efficacy Scale - Short Form (Dennis, 2003)
}

Para cada uma das seguintes afirmações, seleccione a resposta que melhor descreve o seu grau de confiança relativamente à amamentação do seu novo bebé. Assinale a sua resposta colocando um círculo à volta do número que melhor descreve a forma como se sente. Não existem respostas certas ou erradas.

$$
1=\text { nada confiante } \quad 2 \text { = pouco confiante } \quad 3=\text { às vezes confiante } \quad 4=\text { confiante }
$$

\begin{tabular}{ccccc}
\multicolumn{2}{c}{5 = muito confiante } \\
Nada & \multicolumn{3}{c}{ Muito } \\
confiante & \multicolumn{3}{c}{ confiante } \\
1 & 2 & 3 & 4 & 5 \\
1 & 2 & 3 & 4 & 5
\end{tabular}

$\begin{array}{ll}1 & \text { Eu consigo sempre perceber se o meu bebé está a receber leite suficiente } \\ 2 & \text { Eu consigo sempre lidar com sucesso com a amamentação, tal como com }\end{array}$

$\begin{array}{lllll}1 & 2 & 3 & 4 & 5\end{array}$ outras tarefas desafiantes

3 Eu consigo sempre amamentar o meu bebé sem ser necessário dar-lhe suplemento

4 Eu consigo sempre garantir que o meu bebé faz uma boa pega durante toda a mamada

5 Eu consigo sempre gerir de forma satisfatória a amamentação

6 Eu consigo sempre gerir a amamentação mesmo que o meu bebé esteja a chorar

7 Eu posso sempre continuar a desejar amamentar

8 Eu consigo sempre sentir-me à vontade a amamentar o meu bebé

$\begin{array}{lllll}1 & 2 & 3 & 4 & 5\end{array}$

$\begin{array}{lllll}1 & 2 & 3 & 4 & 5\end{array}$

$123 \quad 4 \quad 5$

$\begin{array}{lllll}1 & 2 & 3 & 4 & 5\end{array}$

$\begin{array}{lllll}1 & 2 & 3 & 4 & 5\end{array}$

$\begin{array}{lllll}1 & 2 & 3 & 4 & 5\end{array}$ quando estão presentes outros membros da família

9 Eu consigo sempre sentir-me satisfeita com a minha experiência de amamentar

10 Eu consigo sempre lidar bem com o facto de a amamentação poder consumir tempo

11 Eu consigo sempre amamentar o meu bebé esvaziando uma mama antes de passar à outra mama

12 Eu consigo sempre continuar a amamentar o meu bebé em todas as mamadas

13 Eu consigo sempre amamentar o meu bebé quando ele pede

14 Eu consigo sempre identificar quando é que o meu bebé acabou de mamar

12345

12345

12345

12345

12345

12345 